\title{
Mindful awareness of feelings increases neural performance monitoring
}

\author{
Blair Saunders $^{1}$ - Achala H. Rodrigo ${ }^{2} \cdot$ Michael Inzlicht $^{3}$
}

Published online: 8 September 2015

(C) Psychonomic Society, Inc. 2015

\begin{abstract}
Mindfulness has been associated with enhanced performance monitoring; however, little is known about the processes driving this apparent neurocognitive benefit. Here, we tested whether focusing present-moment awareness toward the nonjudgmental experience of emotion facilitates rapid neural responses to negative performance outcomes (i.e., mistakes). In particular, we compared whether directing present-moment awareness toward emotions or thoughts would enhance the neurophysiological correlates of performance monitoring: the error-related negativity (ERN) and the error positivity $(\mathrm{Pe})$. Participants were randomly assigned to either a thought-focused or an emotion-focused group, and first they completed a preinduction go/no-go task. Subsequently, the groups followed inductions that promoted mindful attention toward either thoughts or emotions, before completing a final postinduction go/no-go session. The results indicated that emotion-focused participants demonstrated higher neural sensitivity to errors in the time course of the ERN, whereas focusing on thoughts had no effect on performance monitoring. In contrast, neither induction procedure altered the amplitude of the later Pe component. Although
\end{abstract}

Electronic supplementary material The online version of this article (doi:10.3758/s13415-015-0375-2) contains supplementary material, which is available to authorized users.

Blair Saunders

blairsaunders01@gmail.com

1 Department of Psychology, University of Toronto, 1265 Military Trial, Toronto, Ontario M1C 1A4, Canada

2 Department of Psychological Clinical Science, University of Toronto, Toronto, Ontario, Canada

3 Rotman School of Management, University of Toronto, Toronto, Ontario, Canada our manipulations also induced changes in behavior, the ERN effects remained significant after controlling for performance. Thus, our results suggest that mindfulness meditation boosts early neural performance monitoring (ERN amplitude), specifically through meditation's influence on affective processing.

Keywords Mindfulness $\cdot$ Meditation $\cdot$ Performance monitoring $\cdot$ Neuroscience $\cdot$ Emotion

Mindfulness - attending to present-moment experience in an accepting, nonjudgmental manner-is a topic of widespread interest across multiple sectors of contemporary society. One particular interest to psychological scientists is the influence of this tuning to the present moment on the processes responsible for monitoring emotion, cognition, and action. Although such performance monitoring underlies flexible behavior across a wide array of domains, a particular focus in cognitive neuroscience research has aimed to understand the processes that allow individuals to monitor for errors. Interestingly, enhanced neural reactivity to errors has recently been reported in experienced mindfulness meditators (Teper \& Inzlicht, 2013), suggesting that one benefit conferred by meditative practice is an increased ability to internally monitor the accuracy of ongoing actions. Less understood, however, are the processes through which meditation enhances monitoring. Does mindfulness improve monitoring by generally directing attention to present-moment thoughts and events? Or does mindfulness improve monitoring in particular by increasing nonjudgmental attention to transient affective events, such as mistakes? Motivated by these questions, the primary purpose of the present research was to experimentally differentiate between qualitatively different aspects of present- 
moment awareness as candidate processes underlying mindfulness-based enhancement of performance monitoring.

\section{Dissociable facets of awareness}

As we navigate our daily lives, our sense of presence, identity, and agency is largely defined by the fluctuating contents and focus of the mind. Rather than arising from a unitary capacity, however, self-awareness consists of partially dissociable mechanisms (Farb et al., 2007; Gallagher, 2000). First, selfawareness can reflect the elaboration of thoughts that arise relatively independently from the present-moment sensory experience, feelings, or environmental context (Gallagher, 2000). During the working day, for example, despite being physically situated within an office space, the mind can wander to thoughts of home life, interpersonal relationships, or even more abstract considerations, including broader goals, ideals, and aspirations. Importantly, this narrative-focused form of self-reference extends across time, to reflect on past occasions, anticipate the future, or occur unanchored to any specific temporal event (Farb et al., 2007; Gallagher, 2000).

Contrasting with narrative focus, awareness can also arise from attention directed toward momentary experiences, such as the processing of thoughts, feelings, or sensations arising from immediate environmental, mental, or bodily states. Adopting this experience-focused (Farb et al., 2007) attention in the workplace, for example, might promote awareness of fluctuating office temperature, seating posture, or current affective states. Critically, this concept of tuning in to the present moment without embellishment or elaboration is fundamental to contemporary definitions of mindfulness (Bishop et al., 2004; Kabat-Zinn, 1994), indicating that state manipulations of experience-focused attention can provide a valuable paradigm to investigate the psychological processes underlying mindfulness (Farb et al., 2007). Here, we differentially emphasized momentary awareness of feelings and momentary awareness of thoughts, examining their differential impacts on neural monitoring and cognitive control.

\section{Mindfulness and performance monitoring}

Central to most models of cognitive control is the suggestion that ongoing performance is continually monitored for events that threaten successful goal attainment, such as conflict, errors, or negative feedback (e.g., Botvinick, Braver, Barch, Carter, \& Cohen, 2001; Holroyd \& Coles, 2002). On a neuroanatomical level, this performance-monitoring process is commonly thought to be served by medial prefrontal structures, such as the anterior cingulate cortex (ACC), which subsequently signal to other brain areas (e.g., the lateral prefrontal cortex) the need for increased control after detecting goal- incongruent events (Botvinick et al., 2001; Holroyd \& Coles, 2002).

Pertinent to the present concerns are two established eventrelated potentials (ERPs) related to error monitoring: the errorrelated negativity (ERN) and the error positivity $(\mathrm{Pe})$ (Falkenstein, Hohnsbein, Hoormann, \& Blanke, 1991; Gehring, Goss, Coles, Meyer, \& Donchin, 1993). The ERN is a negative deflection in the response-locked ERP that peaks with a frontocentral topography within $100 \mathrm{~ms}$ of mistakes, and has commonly been localized to a generator within the ACC (e.g., Dehaene, Posner, \& Tucker, 1994). The Pe is a positive-going deflection that arises later after the response (200-400 ms) and is more broadly distributed across centroparietal sites (Falkenstein et al., 1991). Akin to the ERN, the Pe has also been associated with generators within the medial prefrontal cortex (e.g., Herrmann, Römmler, Ehlis, Heidrich, \& Fallgatter, 2004; van Veen \& Carter, 2002); however, caution should be exercised when localizing such broadly distributed ERPs to dipole sources (cf. Ridderinkhof, Ramautar, \& Wijnen, 2009).

Two recent studies have used the ERP method to investigate performance monitoring in mindfulness (Larson, Steffen, \& Primosch, 2013; Teper \& Inzlicht, 2013). In one study, increased ERN amplitudes and reduced Stroop errors were reported in expert meditators relative to a meditation-naïve control group (Teper \& Inzlicht, 2013). In this study, both meditation experience (in years) and self-reported mindful acceptance were correlated with increasing ERN amplitudes. In light of these results, it has been suggested that the ability to monitor internally generated affective states without judgment or elaboration drives the increased performance monitoring in mindfulness (Teper, Segal, \& Inzlicht, 2013). This suggestion is consistent with emerging views of the ERN, which suggest that, in addition to reflecting conflict monitoring (Yeung, Botvinick, \& Cohen, 2004) or reinforcement-learning processes (Holroyd \& Coles, 2002), the ERN at least partially reflects the negative affective valence of erroneous actions (Aarts, De Houwer, \& Pourtois, 2013; Inzlicht \& Al-Khindi, 2012; Weinberg, Riesel, \& Hajcak, 2012).

One recent study using a brief mindfulness intervention, however, returned results that are partially inconsistent with this affective neuroscience account. More specifically, participants who underwent a 15-min mindfulness induction demonstrated significantly reduced $\mathrm{Pe}$ amplitudes relative to a control group who focused on relaxation and lifestyle ethics (Larson et al., 2013). In contrast, the ERN was only numerically_ but not significantly_-larger for the mindfulness group. The Pe has been associated with a heterogeneous range of psychological processes (see Overbeek, Nieuwenhuis, \& Ridderinkhof, 2005, for a review); however, this ERP has been most commonly associated with attention toward the motivational significance of mistakes (Leuthold \& Sommer, 1999; Ridderinkhof et al., 2009) and conscious error 
awareness (cf. Nieuwenhuis, Ridderinkhof, Blom, Band, \& Kok, 2001; Wessel, 2012). Thus, as was noted by Larson, Steffen, and Primosch, the reduction in Pe amplitude observed in their mindfulness group is difficult to reconcile with existing suggestions that mindfulness enhances presentmoment attention and awareness (e.g., Bishop et al., 2004; Jha, Krompinger, \& Baime, 2007; Kabat-Zinn, 1994). To account for the differences between these studies, the authors suggested that changes in mindfulness stimulated by brief inductions might differ qualitatively from those manifest in trait or expert mindfulness (e.g., Teper \& Inzlicht, 2013). Another possibility, however, is that brief mindfulness inductions are more or less effective, depending on the nature of such inductions.

\section{The present study}

In the present study, we developed short induction procedures to further test the influence of mindfulness on performance monitoring. In particular, we compared participants' inhibitory control (go/no-go performance) and neural monitoring (ERN, Pe) both before and after brief inductions that were tailored to focus nonjudgmental momentary awareness toward qualitatively different aspects of mindful experience.

In line with suggestions that emotional attunement drives increased performance monitoring in mindfulness (Teper et al., 2013), our first induction procedure directed presentmoment attention and awareness toward affective experience. Mirroring the nonelaborative nature of mindfulness, participants were instructed to direct attention toward their present emotional experience without judgment. Here, we predicted that this condition would be associated with increased ERN amplitudes after the induction, relative to the preinduction measurements. Additionally, we predicted that this induction would lead to enhanced inhibitory control on the postinduction go/no-go task, following from the enhanced Stroop regulation observed in previous studies of expert mindfulness meditators (Teper \& Inzlicht, 2013).

Second, it is possible that mindful attention to presentmoment events drives improved performance monitoring without necessitating an affective explanation. Indeed, contemporary models of mindfulness equally emphasize enhanced momentary attention toward thoughts and sensations (Bishop et al., 2004; Kabat-Zinn, 1994). Thus, in a second induction procedure participants were guided to direct nonjudgmental momentary awareness toward thoughts rather than feelings. Here, if mindful attention toward thoughts is sufficient to increase performance monitoring, error-related ERPs should also increase after this induction, resulting in no observed differences between our thought-focused and emotionfocused inductions. Crucially, by forming these two groups we do not wish to suggest that thoughts and emotions rely on distinct psychological or neurophysiological mechanisms: Recent reviews have indicated that cognition and emotion are only minimally decomposable (e.g., Barrett, 2006; Inzlicht, Bartholow, \& Hirsh, 2015; Koban \& Pourtois, 2014; Shackman et al., 2011). However, because many such accounts suggest that emotions involve contextualized valuations that vary by both affective valence and intensity (e.g., Barrett, 2006; Gross, 2015; Russell, 2003), our inductions were intended to differentially influence the engagement of this evaluative aspect of information processing.

Third, if brief mindfulness inductions are not powerful to influence ERN amplitudes, we might observe no differences on this component between the pre- and postinduction tasks, regardless of condition. Finally, since one brief mindfulness induction led to reduced Pe amplitudes (Larson et al., 2013), we further analyzed this component in order to provide a comparison with this existing result, and also to provide a more comprehensive overview of early (ERN) and late (Pe) aspects of performance monitoring.

\section{Method}

\section{Participants}

A power analysis using G*Power 3 (Faul, Erdfelder, Lang, \& Buchner, 2007) for mixed-model testing of a within-between interaction for a medium effect size $(d=0.58$; derived from the study by Teper \& Inzlicht, 2013) revealed that we could attain $80 \%$ power with as few as 26 participants. We decided a priori to collect data until the end of the semester, provided that we had at least 40 participants. Forty-nine undergraduate students from the University of Toronto Scarborough provided informed consent and participated in return for course credit. No interim data analyses were conducted prior to the termination of data collection.

Upon entering the experiment, each participant was randomly assigned to either the emotion-focused or the thought-focused group. Eight participants were excluded from the experiment, due to having insufficient error trials $(<6$; see Olvet \& Hajcak, 2009) to compute reliable ERNs (one participant), software malfunction (six participants), or high levels of electroencephalographic (EEG) artifacts (one participant). Thus, 41 participants $\left(M_{\mathrm{age}}=18.9\right.$ years, $S D=2.84 ; 24$ males, 17 females) contributed data to the final behavioral and ERP analyses: emotion-focused $(N=19)$ versus thought-focused $(N=22)$. Importantly, the groups did not differ in age, $t(39)<$ 1 , gender distribution, $\chi^{2}(1, N=41)<1$, or affect-related individual difference factors: alexithymia, $t(39)<1$ (Bagby, Parker, \& Taylor, 1994), or dispositional mindfulness, $t(39)$ $<1$ (Cardaciotto, Herbert, Forman, Moitra, \& Farrow, 2008; see Table 1 for details). 
Table 1 Demographic information for the thought-focused and emotion-focused groups: Mean $(S D)$

\begin{tabular}{lll}
\hline & Group & \\
\hline Scale & Thought $(n=22)$ & Emotion $(n=19)$ \\
Age & $19.3(3.7)$ & $18.5(1.1)$ \\
\% Male & 59.1 & 57.9 \\
Alexithymia & $49.7(9.0)$ & $52.4(8.8)$ \\
Mindfulness & & \\
Awareness & $35.7(5.5)$ & $36.0(5.6)$ \\
Acceptance & $24.8(9.5)$ & $24.4(6.0)$ \\
\hline
\end{tabular}

Alexithymia, score on the 20-item Toronto Alexithymia Scale; Mindfulness, score on the Philadelphia Mindfulness Scale: Awareness and Acceptance subscales.

\section{Procedure}

The experiment was based on a mixed design, such that participants in each induction group contributed data to both the preinduction and postinduction behavioral and ERP analyses (see Fig. 1 for a schematic illustration of the procedure). Consequently, each group's preinduction go/no-go performance provided baseline measures of cognitive control (ERPs and behavior), with the experimental effects of our induction procedures being assessed through comparison with the equivalent postinduction values of these metrics. Thus, rather than including a separate nonmindfulness control group, we had each participant act as his or her own "control" in the present study. We adopted this pre-post protocol primarily to reduce the levels of the between-subjects factors. Such a design is particularly important for ERP studies, given the high degree of variability in the scalp-recorded EEG signals between individuals. More broadly, within-subjects comparisons (i.e., preinduction vs. postinduction) have the additional benefit of achieving greater statistical power with smaller samples than is possible in between-subjects designs.

Upon entering the experiment, individuals were randomly assigned to either the emotion-focused or the thought-focused group, prepared for EEG recording, and seated comfortably at a computer terminal. After supplying some basic demographic information, participants completed a baseline go/no-go task. This task comprised the stimulus letters " $\mathrm{M}$ " and "W" presented centrally in white on a black background. These letters measured approximated $10 \times 12 \mathrm{~mm}$. Participants were instructed to press the central key on a five-button response box whenever the letter " $M$ " was presented ("go" response), and to withhold responding when the target letter was a "W" ("no-go" response). The letter " $\mathrm{M}$ " was presented on $80 \%$ of trials in order to reinforce "go" responding. Targets were presented until response or for a maximum of $500 \mathrm{~ms}$. A response deadline of $500 \mathrm{~ms}$ was employed so as to encourage fast responding, leading to sufficient errors to compute reliable ERNs. A central fixation cross (random duration: 300$700 \mathrm{~ms}$ ) and a blank screen (random duration: 150-300 ms) preceded and followed the target letters, respectively. Consequently, the response-to-stimulus interval varied randomly between 450 and 1,000 ms. After completing 20 practice trials, each participant performed 350 test trials at this preinduction stage. The test trials were divided equally into seven blocks, separated by self-paced rest periods. The instructions equally emphasized speed and accuracy, asking participants to "Respond as fast and accurately as possible" throughout go/no-go task performance.

Next, participants underwent one of two induction procedures designed to create either an emotion-focused or a

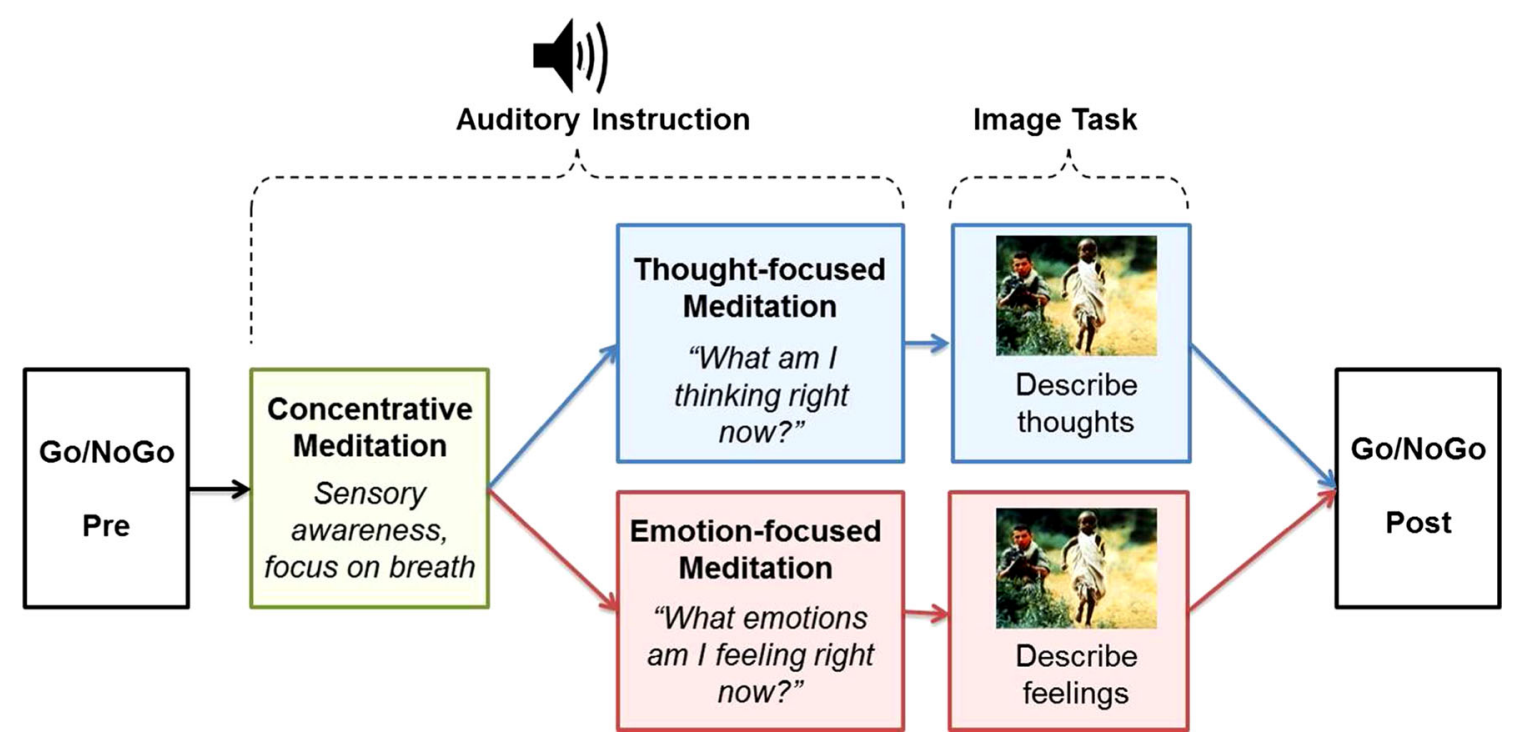

Fig. 1 This schematic depicts our experimental protocol. Induction stages that were specific to the emotion-focused and thought-focused groups are depicted in separate tracks of different colors. 
thought-focused mindset. Overall, our induction procedures consisted of two complementary stages: auditory instructions followed by an image/essay task. This strategy was deliberately chosen to first introduce the participants to mindfulness meditation (auditory task) and then to have them process and attend to a subsequent event using this mindset (image task). As such, participants were asked to maintain the mindsets introduced in the auditory task during the image task, and both tasks were designed to complement each other.

Initially, participants completed brief auditory induction procedures lasting approximately $13 \mathrm{~min}$. Onscreen information prompted the participants to closely follow audiorecorded instructions presented by speakers at their computer terminal. The initial $9 \mathrm{~min}$ of the audio instructions were identical for both groups and aimed to increase sensory awareness using a procedure based on concentrative meditation practice. Initially, participants were instructed to settle into their environment, taking a few moments to look around the room and find a comfortable seating position. After closing their eyes, all participants were guided through a set of exercises directing their attention toward sensory experience. For example, participants were asked to "Bring your awareness to your body, focus your attention on sensations of touch or pressure on your body as it makes contact with the chair." Subsequently, the participants were asked to be mindful of the physical sensations associated with breathing (e.g., observing the movement of the abdomen or the natural pauses between inhalation and exhalation). Throughout this procedure, individuals were instructed to be aware of their experience in the present moment without attempting to change or shape their sensations. In cases in which participants noticed their mind wandering from the concentration exercise, they were instructed that this was "perfectly OK, it is simply what minds do," and that they should subsequently resume focus on the breath.

After these concentration exercises, the audio instructions diverged for the two groups: The emotion-focused instructions were designed to facilitate momentary awareness of feelings in the mind and body, whereas the thought-focused condition directed participants to attend to the information content of the thoughts running through their mind at the present moment. Although these manipulations differed in the aspects of experience that participants were directed toward, the actual auditory instructions given to participants followed a similar structure. Note that, below, italicized words represent divergent instructions between the groups. Participants were instructed to "take a moment to focus on your feelings and emotion [thoughts]. Ask yourself, what emotions am I feeling [what are the thoughts that are going through my mind] right now?" Participants were instructed that there was no need to try to control their experiences, but to "simply be aware of your emotions [thoughts] as they occur, as if you would watch clouds pass by in the sky." Reflecting embodied aspects of emotional experience, participants in the emotion-focused group were also instructed to consider where they were experiencing their emotions in the body. The instructor further prompted the participants to be aware and accepting of their emotions or thoughts, without trying to "fix" anything or achieve any particular state. In the case of mind wandering, participants were instructed "when your mind wanders, simply experience your feelings and emotions [thoughts] as they occur, and using your breath as an anchor, gently reconnect with the here and now." Thus, in both conditions, participants were prompted to foster a mindset that was focused specifically on present-moment experiences.

After these audio instructions, participants were informed to maintain their focus on their "emotions [thoughts]" as they performed a subsequent image-based task. This image task was intended to further reinforce the mindsets introduced during the latter portion of the meditation session. Four emotional images taken from the International Affective Picture System (Bradley \& Lang, 2007) were presented to each participant. The images comprised one picture apiece for four common negative emotions (anger, disgust, fear, and sadness) and measured 1,024×768 pixels. Each image was presented onscreen for a fixed duration of $15 \mathrm{~s}$, followed by open-ended questions with a 1-min fixed response time (RT). Emotion-focused participants were first asked to report their feelings in relation to the picture, and then to report where they experienced these feelings in the body. In contrast, the thought-focused participants were asked to report their thoughts in reaction to the image and subsequently to write about the importance of these thoughts.

After completing these induction procedures, participants completed the state Mindful Attention Awareness Scale (MAAS; Brown \& Ryan, 2003) to test for differences in mindfulness between the conditions. Subsequently, individuals completed a second, postinduction session of the go/no-go task with a procedure identical to that used in the preinduction test.

\section{EEG recording}

Continuous EEG activity was recorded from $11 \mathrm{Ag} / \mathrm{AgCl}$ sintered electrodes embedded in a stretch-Lycra cap. The scalp-electrode montage consisted of midline electrode sites ( $\mathrm{FPz}, \mathrm{Fz}, \mathrm{FCz}, \mathrm{Cz}, \mathrm{CPz}, \mathrm{Pz}$, and $\mathrm{Oz}$ ) referenced to the average activity recorded at the bilateral earlobes. Vertical electrooculography (VEOG) was monitored using a supra- to suborbital bipolar montage surrounding the right eye. During recording, impedances were monitored $(<5 \mathrm{k} \Omega)$ and the EEG signal was digitized at $512 \mathrm{~Hz}$ using ASA acquisition hardware (Advanced Neuro Technology, Enschede, the Netherlands). Offline, the data were band-pass filtered (0.1 to $15 \mathrm{~Hz}$ ) and corrected for eye blinks using regressionbased procedures (cf. Gratton, Coles, \& Donchin, 1983). 
Automatic procedures were employed to detect and reject EEG artifacts. The criteria applied were a voltage step of more than $25 \mu \mathrm{V}$ between sample points, a voltage difference of $150 \mu \mathrm{V}$ within $200-\mathrm{ms}$ intervals, voltages above $85 \mu \mathrm{V}$ and below $-85 \mu \mathrm{V}$, and a maximum voltage difference of less than $0.05 \mu \mathrm{V}$ within $100-\mathrm{ms}$ intervals. These intervals were rejected on an individual-channel basis in order to maximize data retention.

The continuous EEG was segmented into epochs that commenced $200 \mathrm{~ms}$ before the response and lasted for $800 \mathrm{~ms}$ (baseline correction $=-150$ to -50 preresponse). Error- and correct-related ERPs were averaged separately for each experimental condition. Early error-related ERPs (correct-related negativity [CRN], ERN) were operationalized as the amplitude distance between the maximum negative peak following ( 0 to $120 \mathrm{~ms}$ ) and the positive peak preceding $(-80$ to $-20 \mathrm{~ms})$ the response at electrode $\mathrm{FCz}$. The later error positivity $(\mathrm{Pe})$ was operationalized as the mean amplitude $200-400 \mathrm{~ms}$ after mistakes at an electrode. In line with convention, we analyzed at the parietal electrode $\mathrm{Pz}$.

\section{Data analyses}

The behavioral and ERP analyses were submitted to repeated measures analyses of variance (ANOVAs), with simpleeffects comparisons being used to investigate significant interactions. Partial eta-squared $\left(\eta_{\mathrm{p}}{ }^{2}\right)$ provides the effect sizes for all ANOVA statistics, and $95 \%$ confidence intervals (CIs) were used to provide interval estimates for all point-estimate effect sizes derived from differences between the means (e.g., ANOVA main effects or simple-effect comparisons). Only CIs that did not span zero were interpreted as meaningful differences between the point estimates.

\section{Results}

\section{Manipulation check: Text analysis}

Scores on the state MAAS range from 0 (lower levels of state mindfulness) to 6 (higher levels of state mindfulness; Brown \& Ryan, 2003). In our samples, scores on the state MAAS did not differ between groups after the emotion-focused $(M=2.70$, $S D=0.92)$ and thought-focused $(M=2.22, S D=1.14)$ inductions, mean difference $=-0.48,95 \%$ CI $[-1.14,0.18]$. Thus, we found no evidence that our manipulations differentially affected overall mindful attention or awareness.

Next, we used the Linguistic Inquiry and Word Count 2007 (LIWC) software to analyze the open-ended responses obtained during the image task. LIWC is a computerized text analysis program that counts words in any given text into psychologically homogeneous categories (Pennebaker, Chung, Ireland, Gonzales, \& Booth, 2007). We compared the written responses between groups on seven LIWC categories (italicized), covering affective language (positive and negative words); self- versus other-directed attention (self-referential and social words); use of cognitive processing (cognitive words); the percentage of articles; and the use of words with more than six letters (bigwords). Importantly, because we were interested in differences in overall language usage and tone between groups, rather than in the specific responses to each image or question, we submitted the total written output generated by each participant across all questions to LIWC analyses. The written data from two participants (one thought-focused and one emotion-focused) were not included in the LIWC analyses because $\leq 2$ essay responses were recorded for these participants.

Table 2 provides a summary of the group means from the LIWC analyses. The emotion-focused group used on average $4.9 \%$ more self-referential words, $95 \% \mathrm{CI}[3.1,6.8]$, and $3.3 \%$ fewer social words, $95 \%$ CI $[1.5,5.1]$, than the thoughtfocused group. Furthermore, the open-ended responses included on average $2.3 \%$ more negative words for the emotionfocused group, $95 \% \mathrm{CI}[1.3,3.4]$. Together, these results suggest that our manipulation successfully produced a higher degree of emotional, self-referential processing in the emotionalfocused than in the thought-focused group. Furthermore, participants in the thought-focused group used on average $4 \%$ more cognitive words than those in the emotion-focused group, $95 \%$ CI $[0.8,5.3]$. Finally, thought-focused participants on average used 291 more words than the participants in the emotion-focused group, $95 \%$ CI [190.6, 391.5]. We observed no reliable differences between the percentages of positive words used between groups, mean difference $=0.2 \%, 95 \%$ CI $[-0.3,0.6]$, or the average usage of "big words" ( $>6$ letters), mean difference $=2.2 \%, 95 \% \mathrm{CI}[-3.2,7.5]$.

Table 2 Summary of the Linguistic Inquiry and Word Count 2007 (LIWC) software's output for the essay questions

\begin{tabular}{lll}
\hline & Group & \\
\hline LIWC Word Category & Thought & Emotion \\
\% Self-references (I, me, my) & $7.1(2.2)$ & $12.0^{* * *}(3.5)$ \\
\% Social (talk, they, child) & $6.2(3.0)$ & $2.9^{* *}(2.5)$ \\
\% Positive (love, nice, sweet) & $1.2(0.7)$ & $1.1(0.8)$ \\
\% Negative (hurt, ugly, nasty) & $2.1(1.2)$ & $4.4^{* * *}(2.0)$ \\
\% Cognitive (cause, know, ought) & $11.0(2.6)$ & $7.0^{* *}(4.2)$ \\
\% Articles (a, an, the) & $7.3(1.9)$ & $7.6(2.7)$ \\
\% Big words (>6 letters) & $17.7(3.7)$ & $19.9(11.5)$ \\
Mean word count & $449.5(198.3)$ & $158.4^{* * *}(74.6)$
\end{tabular}

Percentages of word usage (with standard deviations), LIWC word categories (with example words), and the mean overall word counts are presented separately for the narrative- and experience-focused groups. ${ }^{* *} p<.01,{ }^{* * *} p<.001$ (independent-samples $t$ test, equal variances assumed). 


\section{ERP data}

Our analyses focused on difference ERPs $(\triangle E R N=E R N$ minus $\mathrm{CRN} ; \triangle \mathrm{Pe}=\mathrm{Pe}$ minus equivalent activity on correct trials) in order to unambiguously assess the sensitivity of neural monitoring processes to performance accuracy (Luck, 2005). To provide a comprehensive account of the data, however, traditional ERP analyses (i.e., including the factor Trial Type) are also summarized. ERP amplitudes were subjected to a repeated measures ANOVA including the factors Time Point and Group. The $95 \%$ CIs for the differences between means are presented for ANOVA main effects and the simpleeffects comparisons.

ERN amplitude Analyses of the $\triangle \mathrm{ERN}$ (see Fig. 2, right panels) revealed a significant interaction between time point and group, $F(1,39)=6.241, p=.015, \eta_{\mathrm{p}}{ }^{2}=.141$, but no main effect of time point, $F(1,39)=1.972, p>.10,95 \% \mathrm{CI}[-2.51$, $0.41] .{ }^{1}$ Although the $\triangle \mathrm{ERNs}$ did not differ between the preinduction $(M=-14.43 \mu \mathrm{V}, S D=6.83)$ and postinduction $(M=$ $-13.60 \mu \mathrm{V}, S D=6.30)$ measures for the thought-focused group, $F<1,95 \%$ CI $[-1.20,2.86]$, the magnitude of the $\triangle \mathrm{ERN}$ was more negative for the emotion-focused group after concentration on feelings $(M=-16.80 \mu \mathrm{V}, S D=6.47)$ than in the preinduction test $(M=-13.90 \mu \mathrm{V}, S D=5.88), F(1,39)=$ $7.226, p=.011, \eta_{\mathrm{p}}{ }^{2}=.156,95 \%$ CI $[-5.08,-0.72]$. In contrast, comparisons did not reveal differences between the groups on the $\triangle \mathrm{ERNs}$ before, $F<1,95 \%$ CI $[-3.52,4.59]$, or after, $F(1$, $39)=2.554, p=.118, \eta_{\mathrm{p}}{ }^{2}=.061,95 \%$ CI $[-7.23,0.85]$, the induction procedures.

Next, we investigated the ERN results, including the effect of trial type (see Fig. 2, left panels). Here, ERP amplitudes were more negative for errors $(-10.81 \mu \mathrm{V}, S D=7.01)$ than for correct trials $(-1.32 \mu \mathrm{V}, S D=11.93), F(1,39)=111.82$, $p<.001, \eta_{\mathrm{p}}{ }^{2}=.741$. We also observed a significant three-way interaction between group, time point, and trial type, $F(1,39)$ $=4.177, p=.048, \eta_{\mathrm{p}}{ }^{2}=.10$. However, simple-effects comparisons investigating the Group $\times$ Time Point $\times$ Trial Type interaction did not reveal differences on the ERN or CRN for any between-group (emotion-focused vs. thought-focused) or within-group (pretest vs. posttest) comparison (all $p \mathrm{~s}>.05$ ). No other main effects or interactions were statistically significant, all $F \mathrm{~s}>1.771$, all $p \mathrm{~s}>.10$, all $\eta_{\mathrm{p}}{ }^{2} \mathrm{~s}<.043$.

\footnotetext{
${ }^{1}$ This interaction was also significant if we operationalized the ERN as the mean amplitude from 0 to $100 \mathrm{~ms}$ following an error, $F(1,39)=6.526$, $p=.015, \eta_{\mathrm{p}}{ }^{2}=.143$. Follow-up simple-effects tests revealed that this was due to an increased $\triangle E R N$ amplitude for the emotion-focused group in the postinduction $(M=-8.44 \mu \mathrm{V}, S D=1.03)$ relative to the preinduction $(-6.27 \mu \mathrm{V}, S D=1.02), F(1,39)=5.077, p=.03, \eta_{\mathrm{p}}{ }^{2}=.115$. In contrast, we found no significant difference between time points for the thoughtfocused group, $F(1,39)=1.764, p=.192, \eta_{\mathrm{p}}{ }^{2}=.043$. Thus, our effects were not dependent on our selection of a method to operationalize the ERN.
}

Pe amplitude Analyses of the $\Delta \mathrm{Pe}$ at electrode $\mathrm{Pz}$ did not reveal a significant interaction between group and time point, $F(1,39)=0.429, p=.516, \eta_{\mathrm{p}}{ }^{2}=.011$, suggesting that our mindfulness inductions did not differentially affect the amplitude of this later correlate of performance monitoring. The main effect of time point was also not significant, $F(1,39)=0.055, p=.815, \eta_{\mathrm{p}}{ }^{2}=.001$; see Fig. 3 (right panels). When we analyzed the data in a second model including the main effect of trial type (correct ERP, error ERP), we found more-positive amplitudes for errors $(M=$ $9.29 \mu \mathrm{V}, S D=6.01)$ than for correct responses $(M=$ $-1.91 \mu \mathrm{V}, S D=3.39), F(1,39)=106.524, p<.001$, $\eta_{\mathrm{p}}{ }^{2}=.732$. However, the critical interaction between time point, group, and trial type was not significant, $F(1,39)=$ $0.429, p=.516, \eta_{\mathrm{p}}{ }^{2}=.011$. No other main effects or interactions were statistically significant; see Fig. 3.

Visual inspection of Fig. 3 also suggests that the Pe amplitude might have peaked earlier after the emotion-focused induction relative to the preinduction baseline. To explore this possibility, we ran an identical ANOVA on the peak latencies of the $\Delta \mathrm{Pe}$ in a search window 200 to $400 \mathrm{~ms}$ after the response. This exploratory analysis revealed that the Pe peaked earlier for the postinduction $(M=290 \mathrm{~ms}, S D=54)$ relative to the preinduction $(M=319 \mathrm{~ms}, S D=47 \mathrm{~ms})$ task, $F(1,39)=$ $15.192, p<.001, \eta_{\mathrm{p}}{ }^{2}=.280$. However, neither the critical interaction between time point and group, $F(1,39)=1.542$, $p=.222, \eta_{\mathrm{p}}{ }^{2}=.038$, nor the main effect of group, $F<1$, was statistically significant.

\section{Behavioral data}

Responses with RTs $<100$ ms were discarded from all analyses. The mean RT measures were submitted to a repeated measures ANOVA with the within-subjects factors Time Point (preinduction vs. postinduction) and Trial Type (Correct "go" response RT vs. false alarm [incorrect "nogo"] RT) and the between-subjects factor Group (focus emotion vs. focus thought). No-go error rates were submitted to a similar repeated measures ANOVA excluding the Trial Type factor. The $95 \%$ CIs for the difference between means are presented for the ANOVA main effects and the simpleeffects comparisons.

RTs As is often reported in go/no-go paradigms, the mean RT on error trials $(M=261 \mathrm{~ms}, S D=28)$ was faster than that for correct trials $(M=304 \mathrm{~ms}, S D=32), F(1,39)=454.957$, $\eta_{\mathrm{p}}{ }^{2}=.921, p<.001,95 \%$ CI [39, 48]. Furthermore, group interacted with time point, $F(1,39)=27.784, p<.001$, $\eta_{\mathrm{p}}{ }^{2}=.416$. Simple-effects comparisons revealed that postinduction performance $(M=276 \mathrm{~ms}, S D=28)$ was faster than preinduction performance $(M=290 \mathrm{~ms}, S D=32)$ for the thought-focused group, $F(1,39)=16.416, p<.001, \eta_{\mathrm{p}}{ }^{2}=.296$, $95 \%$ CI $[7,21]$. In contrast, emotion-focused participants 
A Emotion-focused Group

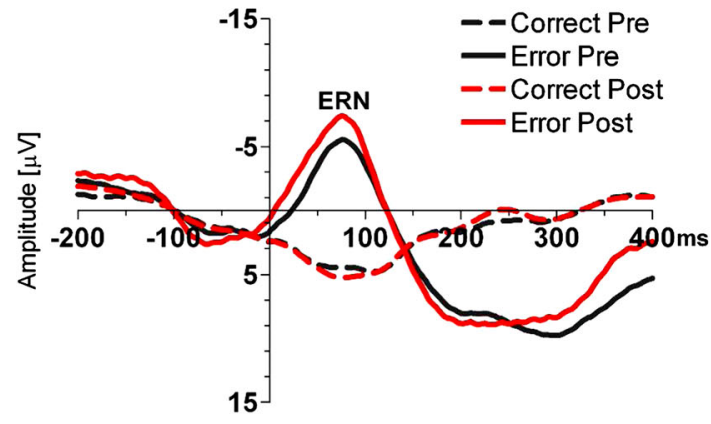

B Thought-focused Group

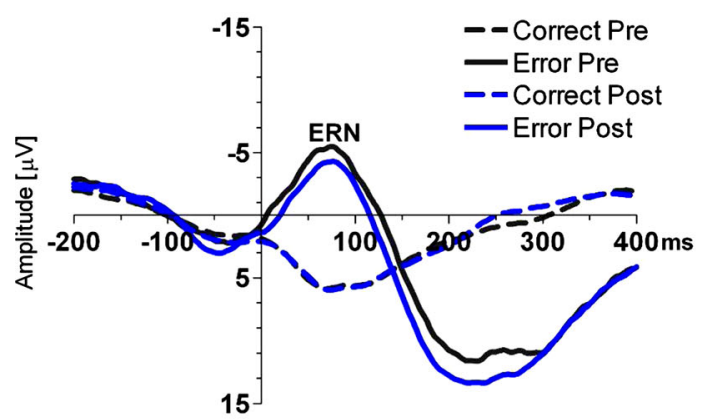

Fig. 2 Error-related negativity (ERN) waveforms: Response-locked event-related potentials (ERPs) are depicted at electrode FCz. The preinduction waveforms are depicted in black, and the potentials recorded after the induction are presented in different colors for the

responded more slowly after concentrating on their feelings $(M=289 \mathrm{~ms}, S D=34)$ than before the manipulation $(M=$ $277 \mathrm{~ms}, S D=28), F(1,39)=11.769, p=.001, \eta_{\mathrm{p}}{ }^{2}=.232,95 \%$ CI $[-20,-5]$. Between-group differences were not observed for RTs at either time point (all $95 \%$ CIs included zero). No other main effects or interactions were statistically significant, all $95 \%$ CIs included zero.

Error rates The main effect of time point indicated that participants were more likely to incorrectly respond on a no-go trial at the postinduction $(M=40.2 \%, S D=20.3)$ than at the preinduction $(M=36.4 \%, S D=22.3)$ time point, $F(1,39)=4.094, p=.05, \eta_{\mathrm{p}}{ }^{2}=.095,95 \%$ CI $[0.0$, 7.6]. This main effect further interacted with group, $F(1$, $39)=5.674, p=.022, \eta_{\mathrm{p}}{ }^{2}=.127$. Subsequent comparisons revealed that the thought-focused group made more errors after $(M=44.0 \%, S D=16.3)$ than before $(M=35.8 \%$, $S D=13.8)$ the induction, $F(1,39)=10.470, p<.002$, $\eta_{\mathrm{p}}{ }^{2}=.212,95 \%$ CI $[3.1,13.5]$. Conversely, error rates did not differ between the preinduction $(M=37.0 \%$, $S D=16.7)$ and postinduction $(M=36.3 \%, S D=15.8)$ sessions for participants who were instructed to focus on their emotions, $F<1,95 \%$ CI $[-6.3,4.9]$.

Thus, together the overall pattern of behavioral results indicates that participants who underwent the emotion-
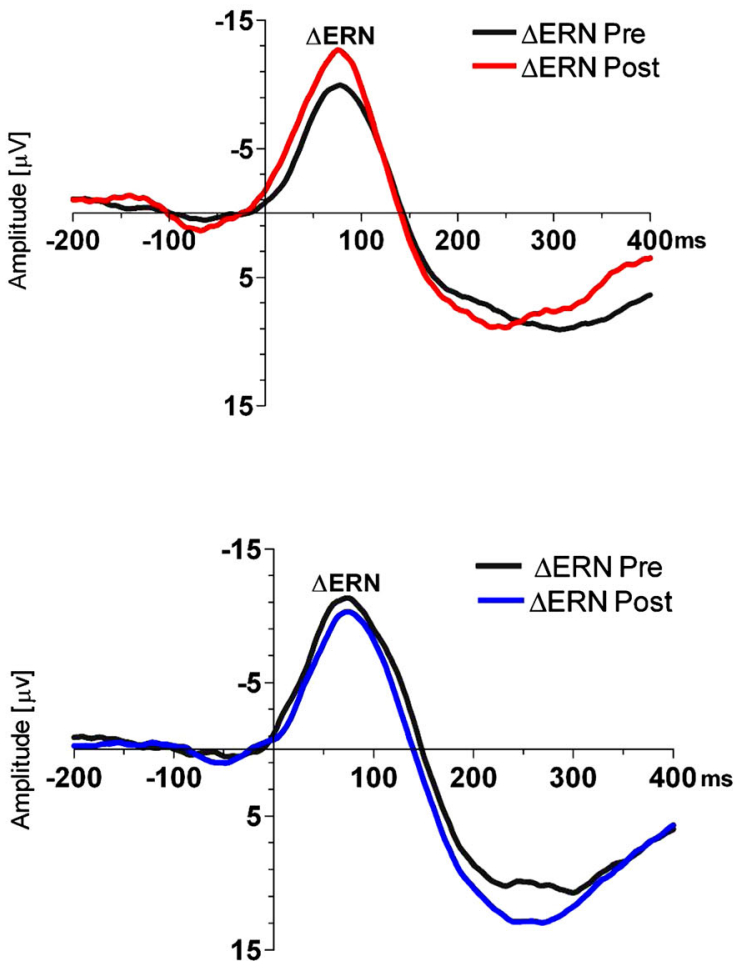

emotion-focused (A) and thought-focused (B) groups. Correct- and error-related ERPs (left panels) and difference ERPs (error activity minus correct activity; right panels) are presented independently.

focused induction performed more slowly after focusing on their feelings, whereas thought-focused participants became less cautious (faster and less accurate) after focusing on their thoughts.

\section{$\triangle E R N$ results controlling for behavioral and LIWC differences between groups}

Because behavioral differences were observed between the groups, it might be argued that our initial group-level ERP effects are confounded by the behavioral effects of the induction procedures. However, indicating that this was not the case, the interaction between time point and group remained significant when controlling for differences in RTs and choice error rates between the pre- and postinduction tasks (e.g., postinduction RT minus preinduction RT) using an analysis of covariance (ANCOVA) model, $F(1,37)=7.562, p=.009$, $\eta_{\mathrm{p}}{ }^{2}=.170$.

Additionally, the LIWC analysis revealed that thoughtfocused participants wrote longer essay responses than participants in the emotion-focused group. Therefore, it could be argued that writing about thinking engaged a more elaborative or ruminative process and, furthermore, that physically typing out these thoughts might have attenuated awareness-related increases in neural monitoring (i.e., those caused by the 
A Emotion-focused Group

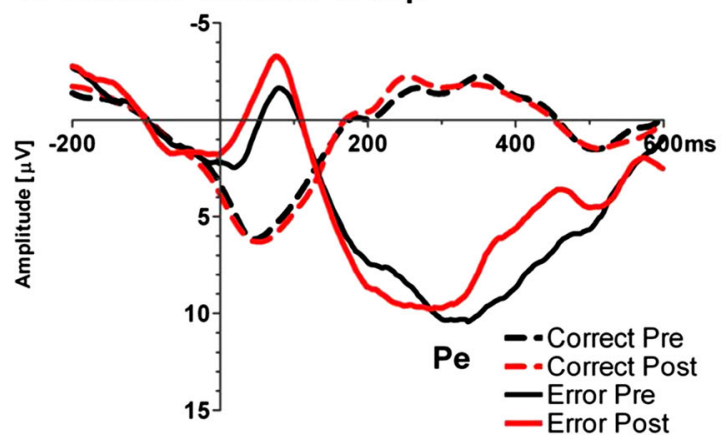

B Thought-focused Group

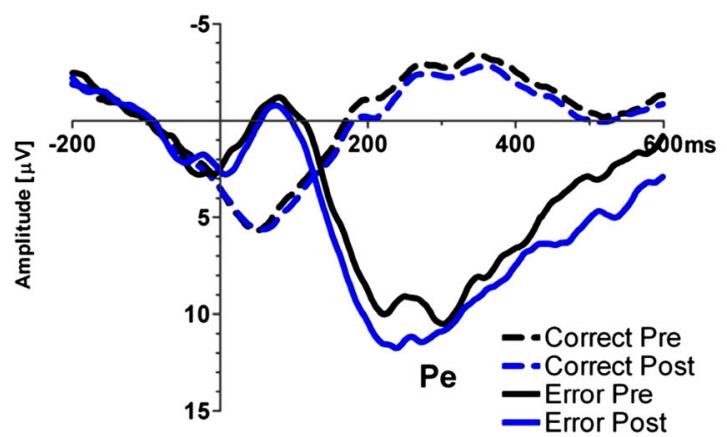

Fig. 3 Error positivity (Pe) waveforms: Response-locked ERPs are depicted at electrode Pz. The preinduction waveforms are depicted in black, and the ERPs recorded after the induction are presented in different colors for the emotion-focused (A) and thought-focused (B)

preceding meditation procedure) by depleting cognitive resources. However, the interaction between time point and group remained significant when controlling for overall word count using the ANCOVA, $F(1,36)=4.558, p=.04, \eta_{\mathrm{p}}{ }^{2}=.112$. In fact, this interaction remained significant when controlling for all LIWC and behavioral differences between groups, $F(1$, $30)=7.251, p=.011, \eta_{\mathrm{p}}{ }^{2}=.195$. $^{2}$

Crucially, these additional analyses indicate that the observed effects of our manipulations on the $\triangle \mathrm{ERN}$ are unlikely to be accounted for by low-level differences between the conditions, such as the content of the essay responses or changes in behavior between the pre- and postinduction tasks. Additionally, because we used a two-stage induction procedure, controlling for the LIWC results suggests that our ERP results were not driven solely by the essay responses to the images.

\footnotetext{
2 The sample contributing to these ANCOVA analyses $(N=39)$ was smaller than that for the initial repeated measures ANOVA due to two participants having insufficient LIWC data to be included in as a covariate (see the Manipulation Check section for details). Importantly, the similar effect sizes observed for the critical interaction (Group $\times$ Time Point) in the original ANOVA $\left(\eta_{\mathrm{p}}{ }^{2}=.141\right)$ and the $\operatorname{ANCOVA}\left(\eta_{\mathrm{p}}{ }^{2}=.112\right.$ or .195) suggest that we observed interactions of similar magnitudes in both groups.
}
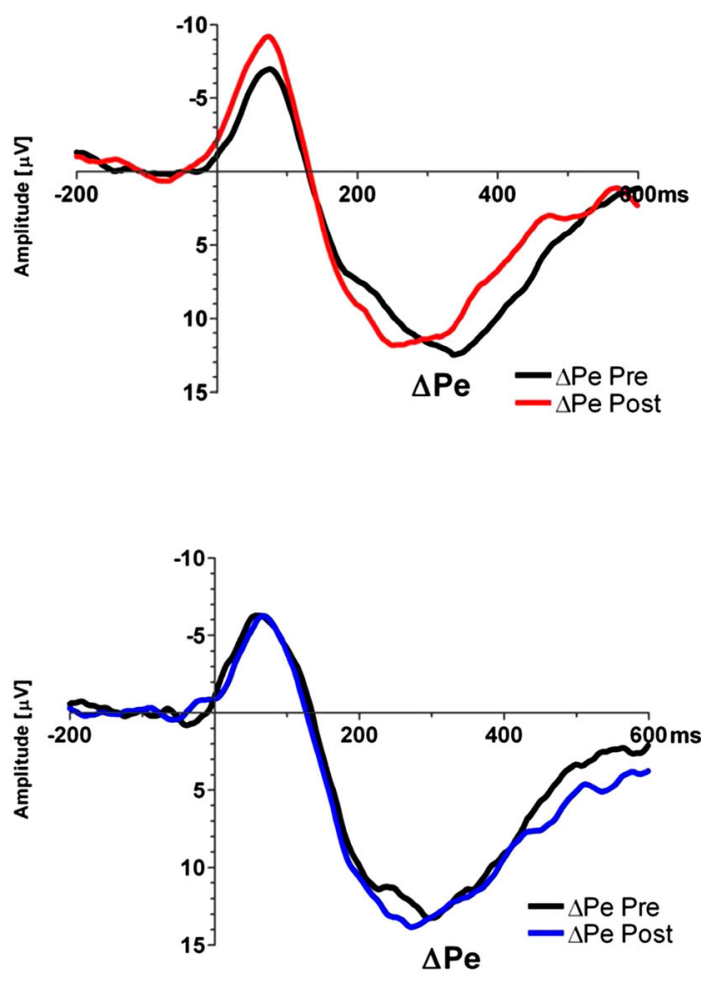

groups. Correct- and error-related ERPs (left panels) and difference ERPs (error activity minus correct activity; right panels) are presented independently.

\section{Discussion}

Our results provide novel evidence linking attunement with momentary affective experience with early neural performance monitoring. Specifically, $\triangle E R N$ magnitude was increased when participants underwent an induction procedure that emphasized being mindful of present-moment feelings. In contrast, the thought-focused induction had no detectable influence on neurophysiological reactivity to errors. Furthermore, neither induction procedure influenced the Pe, a later ERP related to attentional and conscious aspects of error monitoring. Behavioral differences also occurred between the groups: The thought-focused group became faster and less accurate after the induction, whereas the emotionfocused group became significantly slower and numerically more accurate after the postinduction task. Thus, our thoughtfocused and emotion-focused manipulations appear to have differentially influenced levels of response caution on the postinduction go/no-go tasks. It is important to note, however, that our ERP results remained significant after controlling for these effects on RTs and error rates, suggesting that our neural results are not confounded by behavioral differences between the conditions.

We are aware of only one previous investigation of errorrelated ERPs after a brief mindfulness procedure. In their 
study, Larson et al. (2013) observed no ERN differences between participants who followed mindfulness-based breathing exercises relative to controls who focused on relaxation and lifestyle ethics. In contrast, the Pe was reduced in the mindfulness group relative to the controls in that study. Although several methodological differences exist between these two studies, we believe that the specific emphasis on momentary affective experience in our emotion-focused induction uniquely promoted the facet of mindfulness most relevant to early neural monitoring for mistakes. Specifically, we suggest that directing attention to present-moment feelings in the mind and body produced a tonic state of affective receptivity in emotion-focused participants. Furthermore, because erroneous actions are rapidly evaluated as negative events (Aarts et al., 2013; Hajcak \& Foti, 2008; Lindström et al., 2013), this sustained emotional attunement likely sensitized monitoring mechanisms to the primary source of affective arousal during go/no-go performance (i.e., errors). Conversely, neither the mindful-breathing exercises employed by Larson, Steffen, and Primosch nor the thought-focused attention induced in the present study modulated ERN amplitudes, suggesting that brief interventions that do not specifically emphasize affective aspects of mindfulness do not influence this evaluative control process.

Emerging neuroanatomical evidence also points toward the integration of affect and performance monitoring in the frontal midline structures that putatively generate the ERN (Koban \& Pourtois, 2014; Shackman et al., 2011). The ACC in particular is connected both with brain areas implicated in action monitoring and control (e.g., lateral prefrontal cortex and basal ganglia) and with subcortical structures implicated in visceral, autonomic, and affective aspects of information processing (e.g., insular cortex, amygdala, and hypothalamus) (cf. Barbas, 1995). Neuroimaging studies also frequently implicate this medial prefrontal region in emotional processing and performance monitoring (Etkin, Egner, \& Kalisch, 2011; Koban \& Pourtois, 2014; Shackman et al., 2011). Therefore, consistent with the view that the ACC integrates information across multiple domains in order to facilitate adaptive goaldirected behavior (Shackman et al., 2011), it follows that increased momentary awareness directed toward affective experience should boost neural signals emanating from the ACC, such as the ERN (Dehaene et al., 1994; Pourtois et al., 2010).

Our results also endorse recent suggestions that performance monitoring and cognitive control have foundations in emotional processing (Inzlicht et al., 2015). Existing research suggests that monitoring for mistakes involves basic aspects of emotional processing, such as valence judgments (i.e., is the event "good" or "bad" for me?; Aarts et al., 2013) and increased peripheral arousal (e.g., Hajcak, McDonald, \& Simons, 2003; Lindström et al., 2013). Further suggesting that performance monitoring might reflect a negative affective reaction to errors, recent research indicates that the ERN amplitude is reduced by established moderators of emotional experience (Bartholow, Henry, Lust, Saults, \& Wood, 2012; Hobson, Saunders, Al-Khindi, \& Inzlicht, 2014; Inzlicht \& Al-Khindi, 2012). Crucially, the present findings enrich this literature by demonstrating that attending to emotional metaexperiences (i.e., momentary awareness of affect) amplifies early neurophysiological reactivity to mistakes. Affect comprises multiple phenomena, from transient, potentially implicit, affective pangs to full-blown emotions. Thus, it is important to consider what psychological state is represented by the ERN when we consider it to reflect the affective significance of the error. In one recent study, the ERN was sensitive to avoidance motivation (i.e., punishment), but did not predict subjective emotional experience that arose during task performance (e.g., frustration or anxiety; Saunders, Milyavskaya, \& Inzlicht, 2015b). As such, the early ERN might simply reflect valence (positive vs. negative), but not any particular emotional category. In light of this suggestion, it seems parsimonious to suggest that our emotion-focused manipulation enhanced this rapid, yet rudimentary, evaluative process reflected in the ERN.

Although we observed significant behavioral differences between the manipulations, it should be noted that our findings differ from previous reports that mindfulness enhances attentional control (i.e., fewer Stroop errors; Teper \& Inzlicht, 2013). Instead, participants in our emotion-focused condition responded more slowly and became more accurate after the induction, whereas thought-focused participants became faster and less accurate. Thus, it may be suggested that our inductions differentially influenced the extent to which individuals were motivated to avoid errors, rather than resulting in a change in attentional control per se. A number of methodological differences might account for the discrepancy between our behavioral results and those observed by Teper and Inzlicht (e.g., community vs. student sample; Stroop task vs. go/no-go); however, perhaps the most significant difference was in the participants' levels of mindfulness expertise. Whereas the participants in the present study underwent a brief, lab-based mindfulness induction $(<15 \mathrm{~min})$, participants in the earlier cohort had at least 1 year of meditation experience, with an average of over 3 years' practice. Therefore, mindfulness-based changes in attentional control (i.e., reduced error rates without RT costs) might only occur after extended training, whereas our brief inductions appear to have altered motivation, leading participants to discount (thought-focused) or value (emotion-focused) accuracy.

Additionally, we did not find a significant relationship between behavior and the ERN. More specifically, although focusing on thoughts was associated with less cautious responding and emotion focus led to response slowing, these findings were statistically independent from changes in neural monitoring. These results are inconsistent with some studies reporting significant statistical relationships between the ERN 
and performance (e.g., Gehring et al., 1993; Hobson et al., 2014; Inzlicht \& Gutsell, 2007), but other studies have also reported no association between this ERP and behavior (e.g., Gehring \& Fencsik, 2001; Hajcak \& Simons, 2008; Inzlicht \& Al-Khindi, 2012; Strozyk \& Jentzsch, 2012). Thus, whereas the precise relationship between neurophysiological reactivity to errors and the implementation of control remains to be clarified by ongoing research, the present results are most consistent with suggestions that the ERN might inform performance without directly specifying the extent of behavioral adjustment (Gehring \& Fencsik, 2001; Hajcak \& Simons, 2008; Picton, Saunders, \& Jentzsch, 2012).

\section{Limitations and future directions}

The present study should be considered in light of some important limitations. First, in contrast to the emotion-focused group, thought-focused participants became faster and less accurate in the postinduction task, as compared to their baseline. This finding was not predicted and deserves some consideration. Participants in the thought-focused group used more words to describe their experience in the image task; therefore, it might be suggested that this more elaborative form of processing induced a degree of distractibility in these participants, impairing performance on the present task. However, such off-task distraction would predict increased RTs and error rates, whereas our results are more consistent with a reduction in response caution. Thus, it appears that thought-focused participants underwent a shift in motivation, through which they were no longer driven to maintain accuracy in the second task, preferring instead to speed through the task. Interestingly, such a motivational shift has been suggested to underlie so-called ego depletion effects (cf. Baumeister, Vohs, \& Tice, 2007), in which performance deteriorates on a second task after an initial bout of self-control (cf. Inzlicht, Schmeichel, \& Macrae, 2014). Thus, the thoughtfocused group appeared to demonstrate a shift toward impulsive responding that is widely thought to occur in other instances of sequential-task performance similar to that in the present design.

Second, we did not include a neutral condition in our experiment in which participants simply performed the pre- and postinduction go/no-go tasks without an intervening mindfulness manipulation. Therefore, it may be suggested that we are unable to know the natural course of cognitive control throughout our experiment. Although this limitation could be ruled out entirely with a future study, there is good reason to suspect that the observed increase in ERN amplitudes in the emotion-focused group would not occur naturally during an experiment. In particular, existing studies measuring the ERN over time typically observe reduced (Boksem, Meijman \& Lorist, 2006; Inzlicht \& Gutsell, 2007; Wang et al., 2014) or unchanged (Rigoni, Pourtois, \& Brass, 2015) ERN amplitudes as participants progress through a single task or sequential tasks. Thus, the postinduction ERN results observed in the thought-focused group most closely resemble previous results observed in similar paradigms (i.e., the natural course of the ERN), whereas the ERN increases in our emotion-focused group are better attributed to the effects of our induction procedure. In fact, since the emotion-focused group increased monitoring and response caution after the induction, it may be that this manipulation counteracted the motivational shift that appears to have led thought-focused participants to discount accuracy for speed by the second go/no-go task. Nonetheless, we acknowledge that future research will be needed to verify this possibility.

Because performance monitoring was selectively enhanced when participants deliberately attended to emotion, our results are broadly consistent with suggestions that emotion and cognition might not reflect distinct psychobiological mechanisms (e.g., Barrett, 2006; Inzlicht et al., 2015; Russell, 2003; Saunders, Milyavskaya, \& Inzlicht, 2015a; Shackman et al., 2011). This view of emotion, however, might also conclude that our mindfulness inductions falsely dichotomized presentmoment awareness into states of thought-focused and emotion-focused attention. In light of this potential criticism, it should initially be noted that our manipulations were derived from contemporary definitions of mindfulness, which specify momentary awareness of thoughts, feelings, and sensations (Bishop et al., 2004). Nevertheless, we do not suggest that our inductions selectively modified distinct, biologically independent systems that underlie thinking and feeling. Rather, our manipulation likely took advantage of preexisting, lay conceptualizations of emotions and thoughts that we all possess, with the activation of these categories making the emotionality of the current situation/context more or less salient.

Finally, it is necessary to consider the present methodology within the broader science of mindfulness. Mindfulness has been operationalized using various methods, with mindfulness variously being measured as a disposition (Brown, Goodman, \& Inzlicht, 2013; Creswell, Way, Eisenberger, \& Lieberman, 2007), as an acquired skill (Farb et al., 2007; Jha et al., 2007; Teper \& Inzlicht, 2013), or as a psychological state promoted by brief, targeted inductions (Erisman \& Roemer, 2010; Larson et al., 2013). One strength of the present study was that our manipulations uniquely allowed us to differentiate between the influences of different forms of present-moment awareness more easily than could be managed by correlational approaches. Thus, our findings provide a valuable complement to extant cross-sectional investigations (e.g., Teper \& Inzlicht, 2013). A potential limitation of our study is that our participants were still relative novices after only completing our brief, one-shot mindfulness induction. Future longitudinal research could readily address this question by tracking changes in performance monitoring, 
cognitive control, and emotional attunement during more extended mindfulness training. That said, we do find it highly encouraging that even our very brief meditation engendered neural monitoring effects similar to those observed in expert meditators (cf. Teper \& Inzlicht, 2013).

\section{Conclusion}

The findings of the present study propose that directing present-moment awareness toward different aspects of the experiential field has divergent consequences for performance monitoring. In particular, our results indicate that deliberately becoming more sensitive and attentive toward momentary emotional states - but not momentary thoughts more generally-boosts neurocognitive reactivity to erroneous actions. Not only do these results endorse a recently articulated affective neuroscience account of mindfulness (Teper et al., 2013), but they also ratify previous suggestions that ongoing research should closely consider affective aspects of performance monitoring.

\section{References}

Aarts, K., De Houwer, J., \& Pourtois, G. (2013). Erroneous and correct actions have a different affective valence: Evidence from ERPs. Emotion, 13, 960-973. doi:10.1037/a0032808

Bagby, R., Parker, J., \& Taylor, G. (1994). The twenty-item Toronto Alexithymia Scale: I. Item selection and cross-validation of the factor structure. Journal of Psychosomatic Research, 38, 23-32.

Barbas, H. (1995). Anatomic basis of cognitive-emotional interactions in the primate prefrontal cortex. Neuroscience \& Biobehavioral Reviews, 19, 499-510.

Barrett, L. F. (2006). Solving the emotion paradox: Categorization and the experience of emotion. Personality and Social Psychology Review, 10, 20-46. doi:10.1207/s15327957pspr1001_2

Bartholow, B. D., Henry, E. A., Lust, S. A., Saults, J. S., \& Wood, P. K. (2012). Alcohol effects on performance monitoring and adjustment: Affect modulation and impairment of evaluative cognitive control. Journal of Abnormal Psychology, 121, 173-186.

Baumeister, R. F., Vohs, K. D., \& Tice, D. M. (2007). The strength model of self-control. Current Directions in Psychological Science, 16, 351-355. doi:10.1111/j.1467-8721.2007.00534.x

Bishop, S. R., Lau, M., Shapiro, S., Carlson, L., Anderson, N. D., Carmody, J., . . . Devins, G. (2004). Mindfulness: A proposed operational definition. Clinical Psychology: Science and Practice, 11, 230-241.

Boksem, M. A., Meijman, T. F., \& Lorist, M. M. (2006). Mental fatigue, motivation and action monitoring. Biological Psychology, 72, 123-132.

Botvinick, M. M., Braver, T. S., Barch, D. M., Carter, C. S., \& Cohen, J. D. (2001). Conflict monitoring and cognitive control. Psychological Review, 108, 624-652. doi:10.1037/0033-295X.108.3.624

Bradley, M. M., \& Lang, P. J. (2007). The International Affective Picture System (IAPS) in the study of emotion and attention. In J. A. Coan \& J. J. B. Allen (Eds.), Handbook of emotion elicitation and assessment (pp. 29-46). Oxford, UK: Oxford University Press.

Brown, K. W., Goodman, R., \& Inzlicht, M. (2013). Dispositional mindfulness and the attenuation of neural responses to emotional stimuli. Social Cognitive Affective Neuroscience, 8, 93-99. doi:10.1093/ scan/nss004

Brown, K. W., \& Ryan, R. M. (2003). The benefits of being present: Mindfulness and its role in psychological well-being. Journal of Personality and Social Psychology, 84, 822-848. doi:10.1037/ 0022-3514.84.4.822

Cardaciotto, L., Herbert, J. D., Forman, E. M., Moitra, E., \& Farrow, V. (2008). The assessment of present-moment awareness and acceptance: The Philadelphia Mindfulness Scale. Assessment, 15, 204-223.

Creswell, J. D., Way, B. M., Eisenberger, N. I., \& Lieberman, M. D. (2007). Neural correlates of dispositional mindfulness during affect labeling. Psychosomatic Medicine, 69, 560-565.

Dehaene, S., Posner, M. I., \& Tucker, D. M. (1994). Localization of a neural system for error-detection and compensation. Psychological Science, 5, 303-305. doi:10.1111/j.1467-9280.1994.tb00630.x

Erisman, S. M., \& Roemer, L. (2010). A preliminary investigation of experimentally-induced mindfulness on emotional responding to film clips. Emotion, 10, 72-82.

Etkin, A., Egner, T., \& Kalisch, R. (2011). Emotional processing in anterior cingulate and medial prefrontal cortex. Trends in Cognitive Sciences, 15, 85-93.

Falkenstein, M., Hohnsbein, J., Hoormann, J., \& Blanke, L. (1991). Effects of crossmodal divided attention on late ERP components: II. Error processing in choice reaction tasks. Electroencephalography and Clinical Neurophysiology, 78, 447455. doi:10.1016/0013-4694(91)90062-9

Farb, N. A. S., Segal, Z. V., Mayberg, H., Bean, J., McKeon, D., Fatima, Z., \& Anderson, A. K. (2007). Attending to the present: Mindfulness meditation reveals distinct neural modes of self-reference. Social Cognitive and Affective Neuroscience, 2, 313-322.

Faul, F., Erdfelder, E., Lang, A.-G., \& Buchner, A. (2007). G*Power 3: A flexible statistical power analysis program for the social, behavioral, and biomedical sciences. Behavior Research Methods, 39, 175-191. doi:10.3758/BF03193146

Gallagher, S. (2000). Philosophical conceptions of the self: Implications for cognitive science. Trends in Cognitive Sciences, 4, 14-21.

Gehring, W. J., \& Fencsik, D. E. (2001). Functions of the medial frontal cortex in the processing of conflict and errors. Journal of Neuroscience, 108, 624-652.

Gehring, W. J., Goss, B., Coles, M. G. H., Meyer, D. E., \& Donchin, E. (1993). A neural system for error detection and compensation. Psychological Science, 4, 385-390. doi:10.1111/j.1467-9280.1993. tb00586.x

Gratton, G., Coles, M. G. H., \& Donchin, E. (1983). A new method for off-line removal of ocular artifact. Electroencephalography and Clinical Neurophysiology, 55, 468-484. doi:10.1016/00134694(83)90135-9

Gross, J. J. (2015). The extended process model of emotion regulation: Elaborations, applications, and future directions. Psychological Inquiry, 26, 130-137. doi:10.1080/1047840X.2015.989751

Hajcak, G., \& Foti, D. (2008). Errors are aversive: Defensive motivation and the error-related negativity. Psychological Science, 19, 103108. doi:10.1111/j.1467-9280.2008.02053.x

Hajcak, G., McDonald, N., \& Simons, R. F. (2003). To err is autonomic: Error-related brain potentials, ANS activity, and post-error compensatory behavior. Psychophysiology, 40, 895-903. doi:10.1111/14698986.00107

Hajcak, G., \& Simons, R. F. (2008). Oops! ... I did it again: An ERP and behavioral study of double-errors. Brain and Cognition, 68, 15-21. doi:10.1016/j.bandc.2008.02.118 
Herrmann, M. J., Römmler, J., Ehlis, A. C., Heidrich, A., \& Fallgatter, A. J. (2004). Source localization (LORETA) of the error-relatednegativity (ERN/Ne) and positivity $(\mathrm{Pe})$. Cognitive Brain Research, 20, 294-299.

Hobson, N. M., Saunders, B., Al-Khindi, T., \& Inzlicht, M. (2014). Emotional down-regulation diminishes cognitive control: A neurophysiological investigation. Emotion, 14, 1014-1026. doi:10.1037/ a0038028

Holroyd, C. B., \& Coles, M. G. H. (2002). The neural basis of human error processing: Reinforcement learning, dopamine, and the errorrelated negativity. Psychological Review, 109, 679-709. doi:10. 1037/0033-295X.109.4.679

Inzlicht, M., \& Al-Khindi, T. (2012). ERN and the placebo: A misattribution approach to studying the arousal properties of the errorrelated negativity. Journal of Experimental Psychology: General, 141, 799-807. doi:10.1037/a0027586

Inzlicht, M., Bartholow, B. D., \& Hirsh, J. B. (2015). Emotional foundations of cognitive control. Trends in Cognitive Sciences, 19, 126132. doi:10.1016/j.tics.2015.01.004

Inzlicht, M., \& Gutsell, J. N. (2007). Running on empty: Neural signals for self-control failure. Psychological Science, 18, 933-937.

Inzlicht, M., Schmeichel, B. J., \& Macrae, C. N. (2014). Why self-control seems (but may not be) limited. Trends in Cognitive Sciences, 18, 127-133. doi:10.1016/j.tics.2013.12.009

Jha, A. P., Krompinger, J., \& Baime, M. J. (2007). Mindfulness training modifies subsystems of attention. Cognitive, Affective, \& Behavioral Neuroscience, 7, 109-119. doi:10.3758/CABN.7.2.109

Kabat-Zinn, J. (1994). Wherever you go, there you are: Mindfulness meditation in everyday life. New York, NY: Hyperion.

Koban, L., \& Pourtois, G. (2014). Brain systems underlying the affective and social monitoring of actions: An integrative review. Neuroscience \& Biobehavioral Reviews, 46, 71-84. doi:10.1016/j. neubiorev.2014.02.014

Larson, M. J., Steffen, P. R., \& Primosch, M. (2013b). The impact of a brief mindfulness meditation intervention on cognitive control and error-related performance monitoring. Frontiers in Human Neuroscience, 7, 308. doi:10.3389/fnhum.2013.00308

Leuthold, H., \& Sommer, W. (1999). ERP correlates of error processing in spatial SR compatibility tasks. Clinical Neurophysiology, 110, 342-357.

Lindström, B. R., Mattsson-Mårn, I. B., Golkar, A., \& Olsson, A. (2013). In your face: Risk of punishment enhances cognitive control and error-related activity in the corrugator supercilii muscle. PLoS ONE, 8, e65692. doi:10.1371/journal.pone.0065692

Luck, S. J. (2005). An introduction to the event-related potential technique. Cambridge, MA: MIT Press.

Nieuwenhuis, S., Ridderinkhof, K. R., Blom, J., Band, G. P., \& Kok, A. (2001). Error-related brain potentials are differentially related to awareness of response errors: Evidence from an antisaccade task. Psychophysiology, 38, 752-760.

Olvet, D. M., \& Hajcak, G. (2009). The stability of error-related brain activity with increasing trials. Psychophysiology, 46, 957-961. doi: 10.1111/j.1469-8986.2009.00848.x

Overbeek, T. J., Nieuwenhuis, S., \& Ridderinkhof, K. R. (2005). Dissociable components of error processing: on the functional significance of the $\mathrm{Pe}$ vis-à-vis the ERN/Ne. Journal of Psychophysiology, 19, 319-329.

Pennebaker, J. W., Chung, C. K., Ireland, M., Gonzales, A., \& Booth, R. J. (2007). The development and psychometric properties of the $L I W C 2007$. Retrieved from www. LIWC.net/ LIWC2007LanguageManual.pdf
Picton, L., Saunders, B., \& Jentzsch, I. (2012). "I will fix only my own mistakes": An ERP study investigating error processing in a joint choice-RT task. Neuropsychologia, 50, 777-785. doi:10.1016/j. neuropsychologia.2012.01.011

Ridderinkhof, K. R., Ramautar, J. R., \& Wijnen, J. G. (2009). To PE or not to PE: A P3-like ERP component reflecting the processing of response errors. Psychophysiology, 46, 531-538.

Pourtois, G., Vocat, R., N'Diaye, K., Spinelli, L., Seeck, M., \& Vuilleumier, P. (2010). Errors recruit both cognitive and emotional monitoring systems: Simultaneous intracranial recordings in the dorsal anterior cingulate gyrus and amygdala combined with fMRI. Neuropsychologia, 48, 1144-1159. doi:10.1016/j. neuropsychologia.2009.12.020

Rigoni, D., Pourtois, G., \& Brass, M. (2015). "Why should I care?": Challenging free will attenuates neural reaction to errors. Social Cognitive and Affective Neuroscience, 10, 262-268. doi:10.1093/ scan/nsu068

Russell, J. A. (2003). Core affect and the psychological construction of emotion. Psychological Review, 110, 145-172. doi:10.1037/0033295X.110.1.145

Saunders, B., Milyavskaya, M., \& Inzlicht, M. (2015a). Variation in cognitive control as emotion regulation. Psychological Inquiry, 26, 108-115. doi:10.1080/1047840X.2015.962396

Saunders, B., Milyavskaya, M., \& Inzlicht, M. (2015b). What does cognitive control feel like? Effective and ineffective cognitive control is associated with divergent phenomenology. Psychophysiology, 52, 1205-1217. doi:10.1111/psyp. 12454

Shackman, A. J., Salomons, T. V., Slagter, H. A., Fox, A. S., Winter, J. J., \& Davidson, R. J. (2011). The integration of negative affect, pain and cognitive control in the cingulate cortex. Nature Reviews Neuroscience, 12, 154-167.

Strozyk, J. V., \& Jentzsch, I. (2012). Weaker error signals do not reduce the effectiveness of post-error adjustments: Comparing error processing in young and middle-aged adults. Brain Research, 1460, $41-49$.

Teper, R., \& Inzlicht, M. (2013). Meditation, mindfulness, and executive control: The importance of emotional acceptance and brain-based performance monitoring. Social Cognitive Affective Neuroscience, 8, 85-92. doi:10.1093/scan/nss045

Teper, R., Segal, Z., \& Inzlicht, M. (2013). Inside the mindful mind: How mindfulness enhances emotion regulation through improvements in executive control. Current Directions in Psychological Science, 22, 449-454. doi:10.1177/0963721413495869

van Veen, V., \& Carter, C. S. (2002). The timing of action-monitoring processes in the anterior cingulate cortex. Journal of Cognitive Neuroscience, 14, 593-602. doi:10.1162/08989290260045837

Wang, Y., Yang, L., \& Wang, Y. (2014). Suppression (but not reappraisal) impairs subsequent error detection: An ERP study of emotion regulation's resource-depleting effect. PLoS ONE, 9(e96339), 1-9. doi: 10.1371/journal.pone.0096339

Weinberg, A., Riesel, A., \& Hajcak, G. (2012). Integrating multiple perspectives on error-related brain activity: The ERN as a neural indicator of trait defensive reactivity. Motivation and Emotion, $36,84-100$.

Wessel, J. R. (2012). Error awareness and the error-related negativity: Evaluating the first decade of evidence. Frontiers in Human Neuroscience, 6, 88. doi:10.3389/fnhum.2012.00088

Yeung, N., Botvinick, M. M., \& Cohen, J. D. (2004). The neural basis of error detection: Conflict monitoring and the error-related negativity. Psychological Review, 111, 931-959. doi:10.1037/0033-295X.111. 4.931 